\title{
A Pseudomatched Filter Model Applied to the Transient System of Human Vision
}

\author{
Albertus C. den Brinker, Hans A. L. Piceni, and Frans E. W. Vervuurt
}

\begin{abstract}
A pseudomatched filter configuration is presented as a model for the transient system of the human visual perception. This model has resemblances to the Reichardt $[1]$ and the Rashbass [2] model, but on the other hand the pseudomatched filter model is linear. The choice of the model is based on the shape of the psychophysically measured impulse response and on physiological and functional arguments. The parameters of the model are estimated for different data sets. The behavior of the model agrees with the experimental data in all essential aspects, and can be used for prediction and further modeling of the human visual system.
\end{abstract}

\section{INTRODUCTION}

$\mathrm{A}$ $\mathrm{N}$ important branch in psychophysical research is concerned with the measurement of the characteristics of the visual system at threshold level. Even these small signals are not processed linearly in the visual system, and it is usually assumed that the visual system can be modeled by different channels operating in parallel. In the temporal domain there are usually two channels postulated: the sustained and the transient channel [3]-[6]. Roufs and Blommaert [7] showed that the impulse response of these temporal channels can be measured separately. This paper discusses the parameter estimation of a linear model on the basis of data [7], [8] from impulse responses of the transient channel.

With this type of estimation a compact description of the measurement data is obtained, and predictions on the basis of the model can be made. Of more importance is that models may give insight into the system that is being modeled.

A linear filter configuration is chosen as a model of the transient channel. We prefer to call this model a pseudomatched filter model. A matched filter optimizes the detection performance of the overall system for a certain specified input signal in the presence of white noise at the input of the matched filter. The transient channel is also a detection mechanism in which nonnegligible noise is present, as in all biological systems.

The pseudomatched filter model we propose here is fitted to experimental data. The data on which the parameter estimations are performed are derived from psychophys-

Manuscript received April 10, 1989; revised February 6, 1990. This work was supported by The Board of Directors of Eindhoven University of Technology under Grant CB-53 to the Interdepartmental Group "Retina Models."

The authors are with the Department of Electrical Engineering. Eind hoven University of Technology, NL-5600 MB Eindhoven, The Netherlands.

IEEE Log Number 9144702. ical experiments. These experiments are detection tasks, i.e., a subject states whether he has seen or not seen a certain stimulus. A perturbation technique is used to obtain impulse responses from such experiments. This technique is described by Roufs and Blommaert [7]. Using this technique some a priori information on the impulse response is available. This information must be incorporated into the model that is being fitted, resulting in some restrictions on the degrees of freedom of the chosen model.

The impulse response data 17$\},|8|$ are interpreted as being derived from a continuous system. The model being fitted to these data consists of three linear filters operating in cascade. Two of these filters are designed according to matched filter theory, as will be explained later.

The parameters of this model are determined by minimum least-squares estimation. The objective function. which is being minimized during the estimation process. is a nonlinear function of the parameters of the three filters. For the estimation of the filter parameters standard routines from the NAG library are used [9]-[11].

Threshold-versus-duration curves and de Lange characteristics are predicted by the model (Section VI). In the past nonlinear models [2] [ 12$\}$ have been used to provide a simultaneous explanation of these data. Both the Rashbass model [2] and the model of Kelly and Savoie [12] contain a linear process described by a biphasic impulse response. Such an impulse response is unable to predict the dip in the threshold-versus-duration curves that is found for large field sizes. In both models the nonlinear operator provides the possibility to explain this dip. In contrast, we propose a linear model with an impulse response that directly explains these data. without the need for a nonlinear operator.

It has been shown 17] that the linear model for the transient system proposed by Roufs [4]. 17] and the nonlinear model of Rashbass $[2 \mid$ yield similar behavior for pulselike stimuli. Rashbass [13] showed that these two models can be seen as the extreme cases of a large set of models that are now commonly used for visual processing. We argue that there is a second reason for the quadratic model of Rashbass and the linear model of Roufs to exhibit quite similar behavior. This is done in this paper by showing that a linear interpretation of the measurement data can be found within the class of tilters based on matched filter theory. It is well known that there are strong similarities between matched filtering and autocorrelation processes 
[14] for certain specific input signals. This is explained in more detail in Section III.

The paper is organized in the following way. The data are presented in Section II, and the model in Section III. Section IV describes the nonlinear parameter estimation process and its implementation. The results of this parameter estimation are presented in Section V. Comparisons of predictions of the behavior of the model to experimental data are shown in Section VI. We conclude with a discussion (Section VII).

\section{The Impulse Response Data}

The response of the eye to a certain stimulus is generally supposed to be processed in different channels operating in parallel [3]-[6]. In the temporal domain two channels are usually assumed to intermediate between the physical stimulus and the human percept. These are the sustained and transient channels. The transient channel is tuned to low spatial frequencies and fast temporal changes (cf. [15], [16]).

The available data consist of impulse responses of seven subjects at $1200 \mathrm{Td}$ and four subjects at a $100 \mathrm{Td}$ background [7], [8]. These data are obtained from threshold measurements of flashed disks $\left(1^{\circ}\right.$ visual angle) upon a circular background of the same extent and a certain luminance projected foveally. The surround of the disk is completely dark. It is assumed that in this way the transient channel of the eye is most stimulated [4], [7], and so the measured impulse responses are solely attributable to the transient channel.

The normalized impulse responses were measured with the perturbation technique [7]. The perturbation technique uses a two-pulse temporal excitation, where the response of one pulse (the probe flash with amplitude $\epsilon_{c}$ ) is perturbed by the response of a second (smaller) pulse (the test flash with amplitude $q \epsilon_{c}, q<1$ and delayed by a time $\tau$ ). The detection amplitude $\epsilon_{c}$ is compared to the detection amplitude $\epsilon_{1}$ of a single pulse. The difference in threshold amplitudes $\epsilon_{c}$ and $\epsilon_{1}$ is caused by the test flash. This difference can be determined as a function of the time delay $\tau$ between the two pulses. (Positive $\tau$ means that the disturbing pulse is delayed with respect to the probe pulse.) In this way the shape of the perturbing signal, i.e., the response of the system to the test flash, can be obtained. (For details see [7] and the Appendix.) Several amplitude normalized impulse responses $U_{\delta}^{*}(t)$ have been measured [8]. An example is shown in Fig. 1. The absolute sensitivity of the system to a single flash (the norm factor $N F$ ) can be measured separately. All impulse response data that are used in this paper to estimate the parameters of the pseudomatched filter model have been published previously [8]. For further details on the perturbation technique, see [7].

There are, however, some consequences of this method that we need to discuss with respect to the data obtained. First, the method requires rather time-consuming experiments. For this reason the number of samples (with a dif-

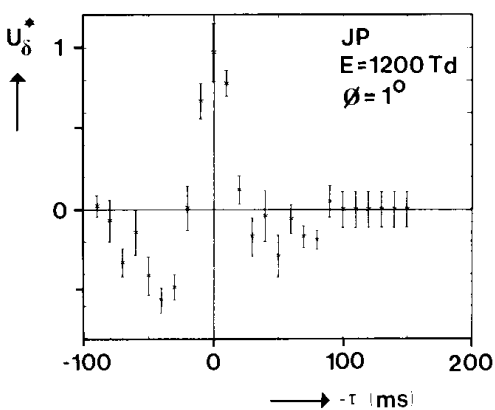

Fig. 1. An example of data obtained from a psychophysical experiment using a perturbation technique, for the measurement of the impulse response. The points represent the mean value, the bars represent twice the standard deviation of the mean. Subject JP, $E=1200 \mathrm{Td}$ and stimulus diameter is $1^{\circ}$

ferent $\tau$ ) that is being measured is as low as possible. The number usually ranges from about 20 to 30 samples. In most cases an extra tail of four or five samples was added to the measured data, with mean values equal to zero and standard deviations taken as the mean of the standard deviations of the actually measured samples. This tail is added in the region where the impulse response is approximately zero to facilitate the estimation process and to prevent severe oscillations of the estimated impulse responses in this region. Secondly, as a result of the perturbation technique the position of the starting point of the impulse response (let us say $t=0$ ) is lost. The origin of the time axis is located exactly on the extremum of the impulse response (see Fig. 1). Note also that the time scale is plotted in the independent variable $-\tau$. We preferred to stick close to the same variable as used in the original measurements, which is $\tau$. To obtain the direction of a normal time axis (later events on the right-hand side) the variable $-\tau$ had to be taken. Thirdly, the extremum should be exactly equal to one. Actual measurement of this extremum gives a value close to but not exactly one, because of the noise in the system (see Fig. 1 at $\tau=0$ $\mathrm{ms}$ ). Since the response axis of the experimental data is normalized, the amplification of our model will not be a free parameter in the estimation process.

The data show that the impulse response is nearly symmetrical with respect to the extremum. This is a wellknown feature of matched filter responses [14]. In the model (see Section III) the impulse responses of the three filters are chosen in such a way that the overall impulse response is approximately symmetrical. As a consequence of the symmetry in the impulse response the phase characteristic of the system and the model will have a large linear component. Independent measurement of the (relative) phase characteristic of the transient visual channel confirms this property [17], [18].

\section{The Pseudomatched Filter Model}

In this section the model is presented. The choice of the model's components is based on the measured data and physiological and functional arguments. This model 


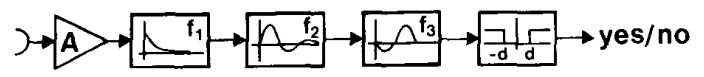

Fig. 2. The pseudomatched filter model, consisting of an amplifier $A$ and three linear filters operating in cascade (for details see text).

has certain similarities to earlier proposed models, notably the Reichardt model [1] and the Rashbass model [2], [13]. This is explained after introduction of the model.

As a model of the transient channel of the visual perception an amplifier $A$ and a cascade of three linear filters $f_{1}, f_{2}, f_{3}$ is proposed (see Fig. 2). The first filter is a firstorder filter with impulse response $f_{1}(t)$

$$
f_{1}(t)=\exp (-a t) H(t)
$$

where $H(t)$ is the Heaviside function

$$
H(t)= \begin{cases}0, & t<0, \\ 1, & t \geq 0 .\end{cases}
$$

The second filter is a second-order filter and its impulse response $f_{2}(t)$ is given by

$$
f_{2}(t)=\exp \left(-b_{1} t\right) \sin \left(\omega_{0} t\right) H(t)
$$

where $b_{1}$ is the damping parameter $\left(b_{1}>0\right)$ and $\omega_{0}$ the angular frequency. The third filter has a biphasic impulse response $f_{3}(t)$, where the second phase is positive and most pronounced

$$
f_{3}(t)=-\exp \left(-b_{2} t\right) \sin \left(\omega_{0} t\right)\{H(t)-H(t-T)\}
$$

where $T=2 \pi / \omega_{0}$ and $b_{2}<0$. This is an exponentially growing sinusoid truncated after one period $T$. Note that if $b_{2}=-b_{1}$ the impulse responses $f_{2}(t)$ and $f_{3}(t)$ are each other's mirrored images with respect to a vertical axis, except for a shift in time, an amplification factor, and the truncation in time

$$
f_{3}(t)=f_{2}(-t+T) \exp \left(b_{1} T\right) H(t), \quad b_{2}=-b_{1} .
$$

Therefore, we will call filter $f_{3}$ pseudomatched on the impulse response of filter $f_{2}$.

The overall impulse response of the three linear filters and the amplifier is called $f(t)$ and is given by a convolution $*$ according to

$$
f(t)=A f_{1}(t) * f_{2}(t) * f_{3}(t) .
$$

Consider a simplification of Fig. 2, where the amplifier and the first filter have been omitted (Fig. 3). Suppose the input signal consists of pulses only, and there is a linear medium $L$ with a second-order impulse response $x(t)=$ $f_{2}(t)$. Suppose further there is a white noise source $n(t)$. If we want to make a detection mechanism, the optimal linear filter is a matched filter [20]. This filter has an impulse response that is a mirrored version of the input signal with respect to the vertical axis. The signal-to-noise ratio $S_{N R}$ apt the output of this (optimal) matched filter would be [14]

$$
\mathrm{SNR}_{\mathrm{opt}}=2 E / N_{0}
$$

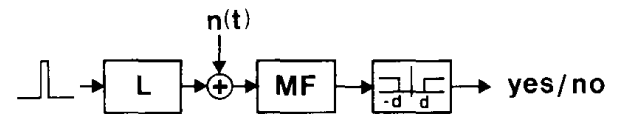

Fig. 3. The matched filter model, consisting of a transmission channel and a matched filter MF (for details see text).

where

$E=\int_{-\infty}^{\infty} x^{2}(t) d t$, the energy of the input signal $N_{0}=$ the spectral density of the white noise signal $n(t)$.

This optimal SNR is not affected by any time delay in the impulse response of the matched filter. This property is used to obtain a matched filter with a causal impulse response. Naturally, this is only possible for signals of finite duration. Since we assumed that $x(t)=f_{2}(t)$ the optimal filter can only approximately be realized. This was done by taking the filter MF (see Fig. 3) with an impulse response $y(t)$ equal to

$$
y(t)=x(T-t) H(t)
$$

which means $y(t)=f_{3}(t)$, under the condition that $b_{2}=$ $-b_{1}$. The signal-to-noise ratio SNR at the output of filter $\mathrm{MF}$ can be calculated and is found to be

$$
\mathrm{SNR}=\mathrm{SNR}_{\mathrm{opt}}\left(1-\exp \left\{-4 \pi b_{1} / \omega_{0}\right\}\right) .
$$

From (9) it can be seen that the signal-to noise ratio is not seriously impaired by the truncation of the mirrored version of $x(t)$ as long as $4 \pi b_{1}>\omega_{0}$. The results from the fits of the overall impulse response $f(t)$ to the measured data (Section IV) are always such that this holds. For instance, with $b_{1}=-b_{2}=30 \mathrm{~s}^{-1}$ and $\omega_{0}=80 \mathrm{rad} / \mathrm{s}$ we find a reduction of only $1 \%$ in the SNR as a consequence of the truncation. Similarly, if $b_{1}$ and $-b_{2}$ have the same order of magnitude (and $b_{1}<\omega_{0} ;-b_{2}<\omega_{0}$ ) the SNR is still close to the optimum [21]. Besides the filters $f_{2}$ and $f_{3}$, Fig. 2 depicts an amplifier and an extra filter $f_{1}$. The amplifier $A$ does not change the SNR, and if the parameter $a$ of filter $f_{1}$ is such that $a>\omega_{0}$ the reduction of the SNR as a consequence of this filter is small [21].

The choice of the filters in Fig. 2 has to be seen in connection with the impulse response data and some physiological findings. First, the measured impulse response as described in Section II is nearly symmetrical round its extremum $\tau=0$. As stated before, this is a feature of matched filter responses. Second, the data are triphasic. By choosing the second and the third filter as above [see formulas (3) and (4)], an approximation to a triphasic impulse response is ensured. As $b_{2}$ is not neccssarily equal to $-b_{1}$, there is some additional freedom to fit the model to the experimental data, with only a small impairment of the SNR [21].

The first filter $\left(f_{1}\right)$ is chosen because it can account for the small asymmetry in the impulse response data. Furthermore, it ensures that the high-frequency falloff is 1.5 log units per octave (with a small ripple caused by the truncation), which agrees well with the de Lange characteristics of stimuli with large spatial extent [22], [23]. 
A magnification factor does not exist in any of the filters; this is separately modeled in the amplifier. As already noted, our data have an extremum exactly equal to one. So this amplification will not be a free parameter when the model is fitted to the impulse response data.

The model is also physiologically inspired. Apart from the amplifier, the ordering in Fig. 2 is such as might be present in the visual system. The first-order filter is an (maybe gross) approximation of a receptor cell response [24]. The second filter is conceived as an approximation to the (linear part of the) response of the retina cells behind the receptor: many physiological measurements show a biphasic impulse response of these cells (cf. [25]). The responses of these cells are transmitted to the cortex and we assume that in this transmission the largest amount of internal noise is introduced (this is illustrated by the noise source $n(t)$ in Fig. 3). Suppose further that the cells in the cortex are functionally interconnected in such a way as to minimize noise influences in their detection operation. Then, under the condition of linearity of the transient channel around threshold level [26], [7], [27], [8], [19], the overall effect of these cortex cells necessarily can be modeled by an impulse response that looks like that of filter 3 . In this way the chain of filters in Fig. 2 is not only chosen to get resemblance to the experimentally determined impulse response (Fig. 1), but it is also related to physiological data (filters 1 and 2) and based on an explicit functional reason (filter 3 ).

From Fig. 3 the similarity in behavior of the Rashbass model and our model can be seen. The convolution of the impulse response of the matched filter and the output of filter $f_{2}$ is similar to an autocorrelation of the output of $f_{2}$ if the input signal is a pulse-like stimulus [19]. Since autocorrelation is a special case of crosscorrelation, the model is reminiscent of the Reichardt model [1]. In the Rashbass model [2] the quadratic element and the integrator with a finite window can be seen as an approximation to an autocorrelation. Therefore, the behavior of the Rashbass model and our linear model will be similar, at least for pulse-like stimuli. Precisely this kind of stimuli are always used to provide data about the transient channel since with low-frequent temporal signals interference of the activity of sustained channels is expected.

\section{The Parameter Estimation Process}

The model outlined in Section III is to be fitted to the impulse response data [7], [8]. From noise analysis of experiments using a perturbation technique it can be shown that the expected value of the noise at the various sampling instants $\tau_{i}$ is approximately equal (see the Appendix). Therefore, the residuals at different $\tau_{i}$ need not be weighted differently in the objective function $\Psi$ (i.e., the function that is to be minimized). The objective function is taken to be the sum of unweighted and squared residuals

$$
\Psi=\sum_{m=1}^{M}\left\{f\left(-\tau_{m}+t_{e x}\right)-U_{\delta}^{*}\left(-\tau_{m}\right)\right\}^{2}
$$

where

$$
\begin{aligned}
M= & \text { number of samples, } \\
\tau_{i n}= & m \text { th sample time, } m=1, \cdots, M, \\
U_{\delta}^{*}\left(-\tau_{m}\right)= & \text { measured (amplitude normalized) re- } \\
& \text { sponse at }-\tau_{m}, \\
t_{c x}= & \text { time of occurrence of the extremum of the } \\
& \text { fitted impulse response } f(t) .
\end{aligned}
$$

This function $\Psi$ depends on the parameters $A, a, b_{1}, b_{2}$, $\omega_{0}$. Since the experimentally determined impulse response is normalized (see Section II) the amplification $A$ is chosen in such a way that the extremum of the impulse response $f(t)$ equals one. As a result $A$ is a function of the other parameters

$$
A=A\left(a, b_{1}, b_{2}, \omega_{0}\right) .
$$

We did not find an analytical expression for this relation. With any parameter set $\left(a, b_{1}, b_{2}, \omega_{0}\right)$ the extremum, which we call $K$, can be searched for numerically. Then $A$ can be set to

$$
A=K^{-1}
$$

and so the maximum is scaled to unity.

We can either search for the maximum of the impulse response $f(t)$, or for zeros of the derivative $d f / d t$. The latter approach was adopted in the computer programs using routine C05AZF from the NAG library [9]. A search for zeros of the derivative was preferred to a search for the maximum, since the former method provides better accuracy in the value of $t_{c x}$. This value $t_{c x}$ is also needed, since the time axis of the measurement data is shifted by this amount (see Section II). Just like $A, t_{e x}$ is a function of the filter parameters $t_{e x}=t_{c x}\left(a, b_{1}, b_{2}, \omega_{0}\right)$. An explicit relation for this function was not found since $d f / d t=0$ holds not only for $t_{c x}$ but for an infinite number of values of $t$.

Although we did not find an analytical expression for $A$, we were still able to formulate first and second derivatives of $f(t)$ explicitly with respect to the parameters. This is possible since a relation between the change in $t_{c:}$ and changes in the parameters can be established. This means that all first and second derivatives of $\Psi$ can be formulated even though a numerical search for $t_{c x}$ (and $A$ ) is performed. This allowed us to use routine E04HEF from the NAG library [9], which is an estimation routine in which first and second derivatives are not approximated by finite differences, but have to be formulated explicitly.

Having implemented the optimization programs in the above-mentioned way, we soon found that the parameter $a$ was ill conditioned in the estimation process. This is a direct consequence of the model: the first-order filter (see Section III) was introduced merely to obtain a sufficient fall-off in the gain characteristic. This means that $a$ is always larger than the other parameters and it has only a minor influence on the shape of the impulse response and is consequently poorly conditioned in the optimization procedure. Therefore, we excluded parameter $a$ from the optimization by setting $a=2 \omega_{0}$. This ensures that the 
influence of this parameter is exactly as outlined above: it affects only the frequencies roughly above the cutoff frequency (under the condition that $b_{1},-b_{2}<\omega_{0}$ ) and has only a minor effect on the shape of the impulse response. In this way the optimization program was restarted, with only three free parameters $b_{1}, b_{2}, \omega_{0}$.

A problem in least-squares optimization is always the possible occurrence of local minima of the function $\Psi$. Fortunately, there is only a small number of parameters (three) and a good guess for the initial parameters can be obtained directly from the experimental data. Twice the width of the positive phase can serve as a first guess of the period time $T\left(=2 \pi / \omega_{0}\right)$. This means about $80 \mathrm{rad} / \mathrm{s}$ for the angular frequency $\omega_{0}$ at the $1200 \mathrm{Td}$ level and about $50 \mathrm{rad} / \mathrm{s}$ at $100 \mathrm{Td}$ for all subjects. Parameters $b_{1}$ and $-b_{2}$ can be chosen equal and roughly estimated from the ratio of positive and negative phase. This is always somewhere between 10 to $30(1 / \mathrm{s})$. With these rough guesses for the initial parameters we never found any local minima, which was checked by trying different starting parameters.

\section{Results of the Estimation Process}

The results of the fit of the model of Fig. 2 to data sets from two different subjects on different background levels are shown in Fig. 4. For most subjects a reasonable fit is obtained. In general, the fits are especially good for the first negative and the positive phase, but poor for the second negative phase. This can also be seen in Fig. 4(a). Note also that the time axis $-\tau$ from Fig. 1 is corrected by $t_{e x}$ so an ordinary time axis $t=t_{e x}-\tau$ is obtained, where $t=0$ indicates the start of the impulse response.

The agreement between experimental data and the fit is shown numerically in Table 1 . In this table the standard deviation $s_{m}$ estimated from the measurements is compared to the standard deviation $s_{e}$ estimated from the result of the fitting procedure. $s_{m}$ is derived from the measured variance averaged over the different samples according to

$$
s_{m}^{2}=\frac{1}{M} \sum_{i=1}^{M} s^{2}\left(\tau_{i}\right)
$$

where $s\left(\tau_{i}\right)$ is the measured standard deviation at the $i$ th sampling moment (see also Fig. 1). The standard deviation $s_{e}$ is estimated from the (final) fit of the model to the measured data according to

$$
s_{c}^{2}=\frac{\Psi}{M-N}
$$

where $\Psi$ is the objective function at the final estimate, $M$ the number of samples, and $N$ the number of degrees of freedom in the estimation [28], [29], [9]. The estimated standard deviation is in most cases larger than the measured standard deviation, reflecting the poor fit of the second negative phase.

The estimated parameters and their variances $\left(s_{i}, i=\right.$ $1,2,3)$ are shown in Table II. The confidence intervals

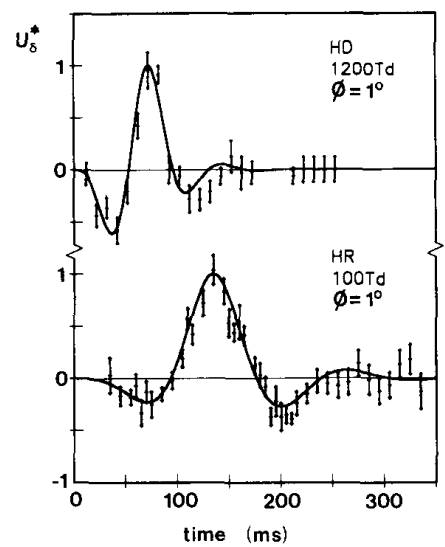

(a)

(b)

Fig. 4. Two examples of estimated impulse responses of the pseudomatched filter model. Model parameters are given in Table II. (a) Subject $\mathrm{HD}, 1^{\circ}$ field at $1200 \mathrm{Td}$. (b) Subject HR, $1^{\circ}$ field at $100 \mathrm{Td}$

TABLE I

Measured Standard Deviation $s_{m}$ and the Standard Deviation $s$ Calculated from the Fit (See Text). The Sum of Squared Residuals is Given by $\Psi$

\begin{tabular}{lcccc}
\hline & $\begin{array}{c}\text { Background } \\
\text { (Td) }\end{array}$ & $s_{\prime \prime}$ & $s_{c}$ & $\Psi$ \\
\hline JR74 & 1200 & 0.120 & 0.149 & 0.488 \\
JAJR & 1200 & 0.086 & 0.129 & 0.364 \\
FB & 1200 & 0.074 & 0.162 & 0.761 \\
JP & 1200 & 0.112 & 0.112 & 0.278 \\
LT & 1200 & 0.139 & 0.130 & 0.369 \\
HD & 1200 & 0.118 & 0.125 & 0.362 \\
IH & 1200 & 0.106 & 0.122 & 0.326 \\
LT & 100 & 0.109 & 0.127 & 0.353 \\
HR & 100 & 0.113 & 0.116 & 0.502 \\
JW & 100 & 0.170 & 0.222 & 1.432 \\
KS & 100 & 0.140 & 0.146 & 0.857 \\
\hline
\end{tabular}

TABLE II

Parameters of the Pseudomatched Filter for Seven Subjects at a 1200 Td Level and Four Subjects at a 100 Td Level (UPPer and Lower Part of the Table, Respectively) In All Cases the Stimulus Diameter is $1^{\circ}$

\begin{tabular}{lccccc}
\hline & $\begin{array}{c}A \cdot N F \\
10^{6}\left(\mathrm{Td}^{-1} \cdot \mathrm{s}^{-5}\right)\end{array}$ & $\begin{array}{c}a \\
\left(\mathrm{~s}^{-1}\right)\end{array}$ & $\begin{array}{c}b_{1} \pm s_{1} \\
\left(\mathrm{~s}^{-1}\right)\end{array}$ & $\begin{array}{c}b_{2} \pm s_{2} \\
\left(\mathrm{~s}^{-1}\right)\end{array}$ & $\begin{array}{c}\omega_{0} \pm s_{3} \\
(\mathrm{rad} / \mathrm{s})\end{array}$ \\
\hline JR74 & 92.5 & 149 & $45.5 \pm 25.6$ & $5.9 \pm 6.4$ & $74.5 \pm 5.6$ \\
JAJR & 74.5 & 179 & $42.9 \pm 19.3$ & $9.5 \pm 6.8$ & $79.5 \pm 5.8$ \\
FB & 50.3 & 181 & $33.4 \pm 14.6$ & $15.4 \pm 9.5$ & $90.4 \pm 7.6$ \\
JP & 66.6 & 167 & $45.3 \pm 18.3$ & $10.5 \pm 6.5$ & $83.7 \pm 5.5$ \\
LT & 64.8 & 169 & $24.3 \pm 7.9$ & $15.1 \pm 10.4$ & $84.6 \pm 5.5$ \\
HD & 207.3 & 181 & $41.5 \pm 17.2$ & $10.5 \pm 8.5$ & $90.7 \pm 6.7$ \\
IH & 25.4 & 174 & $32.4 \pm 11.6$ & $28.5 \pm 13.6$ & $86.9 \pm 7.2$ \\
LT & 1.6 & 110 & $29.3 \pm 11.2$ & $16.0 \pm 6.0$ & $55.1 \pm 4.3$ \\
HR & 0.2 & 100 & $19.6 \pm 4.2$ & $22.4 \pm 6.3$ & $49.9 \pm 2.9$ \\
JW & 2.9 & 117 & $18.9 \pm 7.1$ & $8.0 \pm 6.6$ & $58.5 \pm 4.2$ \\
KS & 1.0 & 100 & $15.8 \pm 7.7$ & $9.7 \pm 8.0$ & $49.9 \pm 4.5$ \\
\end{tabular}

of the parameters can be calculated from the variances [28], [29], [9]. For the true parameter value $\theta_{i}^{\circ}$ the following inequality holds:

$$
\theta_{i}-\sqrt{\operatorname{var} \theta_{i}} t_{\beta / 2, M-N}<\theta_{i}^{\theta}<\theta_{i}+\sqrt{\operatorname{var} \theta_{i}} t_{\beta / 2, M-N}
$$




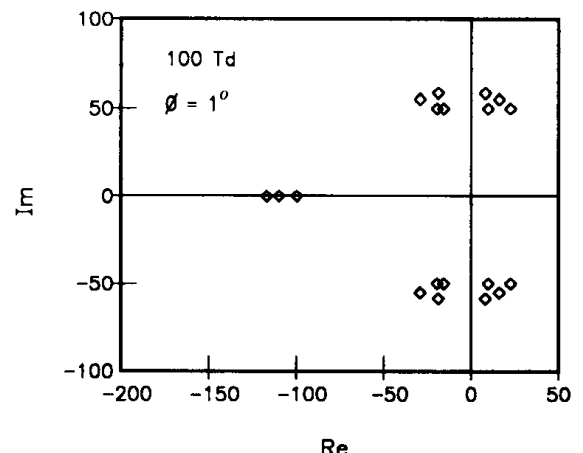

(a)

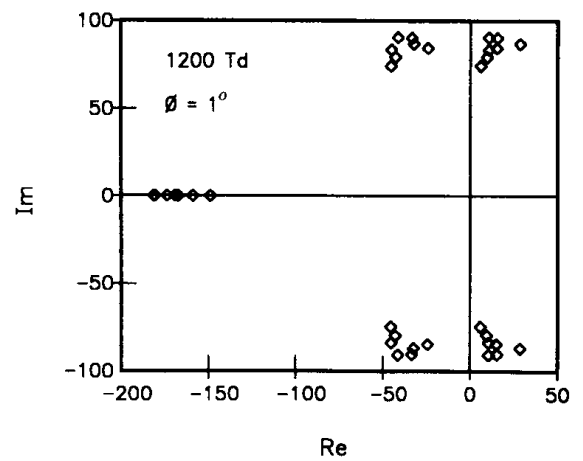

(b)

Fig. 5. The estimated parameters for different subjects of a field of $1^{\circ} \mathrm{di}-$ ameter plotted in the complex plane. (a) Four subjects at 100 and (b) seven subjects at $1200 \mathrm{Td}$ background level.

where

$\theta_{i}=$ the $i$ th estimated parameter, $1 \leq i \leq N$,

$\operatorname{var}\left(\theta_{i}\right)=$ the estimated variance of $\theta_{i}$,

$\theta_{i}^{o}=$ the true value of the $i$ th parameter,

$t_{\beta / 2 . M-N}=100 \beta / 2$ percentage point of the $t$-distribution.

The size of the confidence intervals points out the well and poorly conditioned parameters. This is about the same for all data sets. The frequency parameter $\omega_{0}$ is a very well conditioned parameter; its confidence interval is relatively small (about $10 \%)$. The damping parameters $\left(b_{1}\right.$, $b_{2}$ ) have a (relatively) larger variance.

The estimated parameters are also shown in Fig. 5. From this figure (and Table II) it can be seen that the estimated filter parameters cluster in specific areas in the complex plane, and are fairly independent of the subject (see also the variances in Table II) but strongly dependent on background luminance. From comparison of Fig. 5(a) and (b) we see that only a significant change (given the variances) in the angular frequency parameter $\omega_{0}$ occurs: an increase in frequency with an increase in background level. As a consequence of our assumptions (i.e., $a=$ $2 \omega_{0}$ ) the parameter $a$ changes by the same proportional amount with the background as $\omega_{0}$. There seems to be a trend in the damping parameter $b_{1}$ to higher values at higher levels (see Fig. 5), but this is within the confidence regions (see Table II).
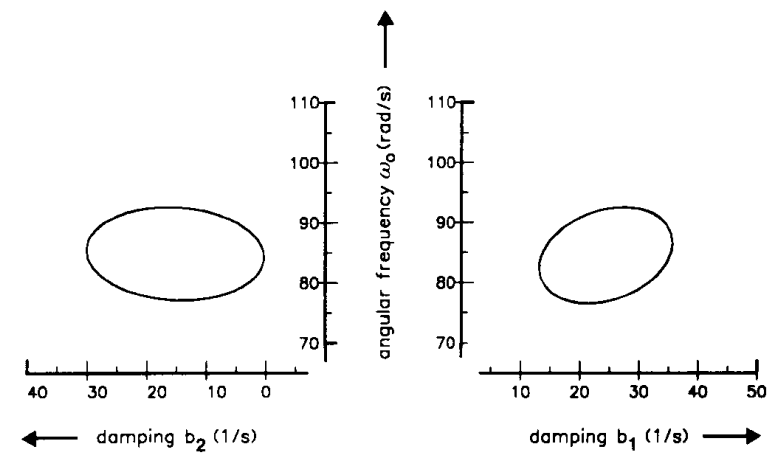

LT 1200Td $\emptyset=1^{\circ}$

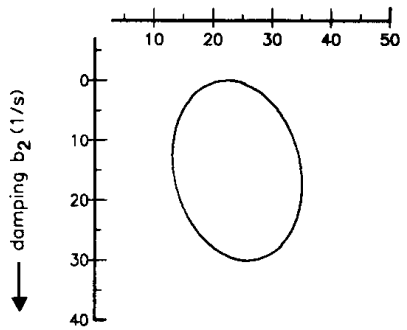

Fig. 6. An example of the estimated indifference regions (see text). Subject LT, $1200 \mathrm{Td}$ and a $1^{\circ}$ field.

In Fig. 6 an example (subject LT, $E=1200 \mathrm{Td}, 1^{\circ}$ field diameter) is shown of the confidence regions of the three estimated parameters. Essentially, the confidence region is a three-dimensional figure, and Fig. 6 shows only the cross sections of this hyper-ellipsoid with the parameter planes. However, Fig. 6 gives a good impression of this three-dimensional hyper-ellipsoid. This was checked by the singular value decomposition (SVD) of the Hessian matrix at the final estimated parameters. The SVD can be used to obtain information on well and poorly conditioned directions in the parameter space [28], [29], [9]. Essentially, the SVD ranges $N$ orthogonal combinations of parameters from best to worst conditioned. In almost all cases the best parameter directions (from the point of view of the estimation process) are approximately the directions of the model parameters. This means that there are no directions in the parameter space with confidence intervals that are much larger than those shown in Fig. 6. This guarantees furthermore that we had a well-defined optimization process, especially since the order of magnitude of the variances of the three estimated parameters is equal (see Table II and Fig. 6).

\section{Vi. Predictions from the Model}

\section{A. Threshold-Versus-Duration Curves}

In Fig. 7 the detection threshold $\epsilon$ is plotted versus the duration $\vartheta$ of a rectangular pulse. The solid line gives the prediction of the model estimated in the previous section for subject JR, at a background level of $1200 \mathrm{Td}$, and a $1^{\circ}$ field without surround. The dots show the measured value of $\epsilon$ for the same conditions [8]. Fig. 7 shows that the predicted curve agrees well with the measured data, 


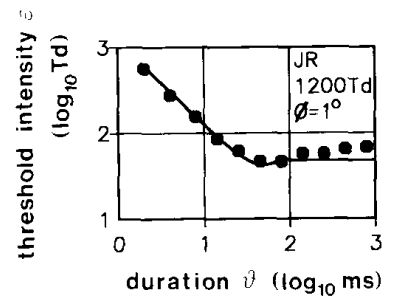

Fig. 7. Prediction and experimental data of a threshold-versus-duration curve. Subject JR, $1200 \mathrm{Td}$ and a $1^{\circ}$ field. Model parameters are given in Table II.

with a small underestimate of the threshold amplitude at long durations. This is a direct consequence of the poor fit of the estimated model to the second negative phase of the experimental data.

\section{B. Frequency Characteristics}

From subject LT estimated impulse responses are available at two different background levels. From these estimated impulse responses the gain characteristics were calculated. The gain characteristics of the estimated linear models are shown in Fig. 8. The figure is to some extent comparable to the de Lange characteristics (amplitude sensitivity versus frequency of a sinusoid, cf. [22], [23]). However, a direct quantitative comparison is not possible. First, we do not have these experimental data of subject LT. Second, we assume that the de Lange curve is an envelope of different channels (cf. [4]) and third, a de Lange curve incorporates stochastic effects (cf. [30]). Nevertheless, Fig. 8 allows a qualitative comparison to the high-frequency side of a de Lange curve. The gain characteristics show that at the higher background level the peak value of the gain curve is lower and the cutoff frequency is higher and that the high-frequency asymptotes of both levels (virtually) coincide. These adaptive effects of the threshold behavior are in agreement with the experimental data [22], [23] on de Lange characteristics, while the slope of the high-frequency asymptote also agrees well with the experimental results.

For subject JP the gain and phase characteristics were measured using a subthreshold summation technique, in the same way as the impulse responses were determined [17], [18]. The advantages of this method are the possibility of measuring the amplitude sensitivity of the transient visual system (i.e., without contributions from other channels), and of revealing the (relative) phase characteristic. This means that a quantitative comparison of the frequency behavior of the estimated linear model with experimental data is possible. We did not allow any vertical shifts in the predicted curve since the model is fully specified by its parameters, including the gain (see Table II), and the contributions of probability summation in the measurement data should only be negligible as a consequence of the perturbation technique.

Fig. 9(a) shows the gain characteristic of the model (continuous line) and the subthreshold measured data (dots) of the amplitude sensitivity of the transient chan-

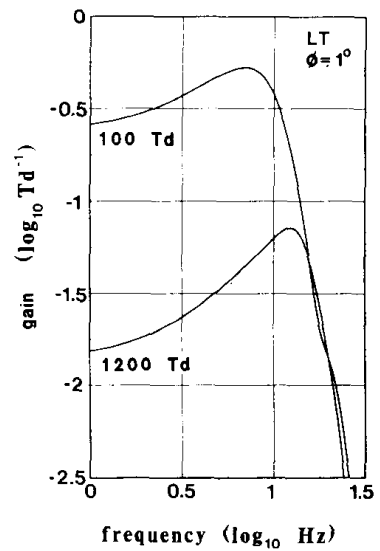

Fig. 8. Gain characteristics of the pseudomatched filter model. Subject LT $1^{\circ}$ field, 100 and $1200 \mathrm{Td}$ background level. Model parameters are given in Table II.

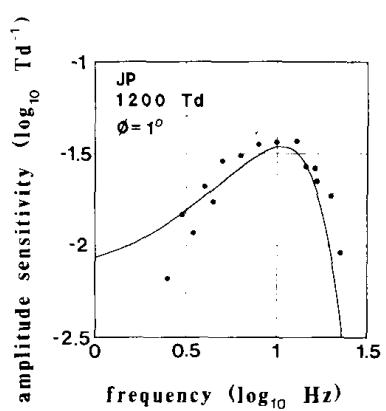

(a)

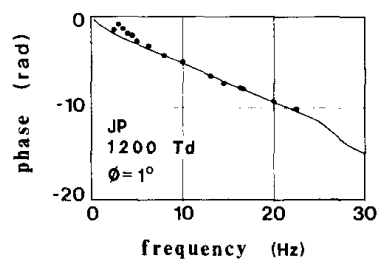

(b)

Fig 9. Gain and phase characteristics of the pseudomatched filter model and experimental data of the subthreshold measurement of these characteristics. Experimental data replotted from [17]. Subject JP, $1^{\circ}$ field at 1200 Td background. Model parameters are given in Table II.

nel. The predicted curve and the experimental data are in good agreement, except for the lowest frequencies. This is again a consequence of the underestimation of the second negative phase of the impulse response; a more pronounced second negative phase results in smaller predicted values of the low-frequency side of the gain characteristic. Fig. 9(b) shows the phase characteristic of the model (continuous line) and the experimental data (dots). Both show a nearly linear dependence on frequency.

\section{Discussion}

In this paper we have presented a model for the threshold behavior of the transient channel of the visual system. 
The model is thereby restricted to describe the processing of stimuli with a low-frequency spatial content and fast changes in temporal wave form [3], [6], [15], [16].

The model consists of a cascade of linear filters, and is based on physiological and functional arguments. These arguments provide a conceptually attractive model. The functional argument, i.e., the fact that the system performs according to a nearly optimal detection unit, is in our view the most important one. However, the choice of the filters might contain weak points: the filter model is somewhat too rigid to contain all details of actual occurring physiological responses. Providing possibilities for further detail in the first and second filters would mean introducing extra parameters. In view of the parsimony principle we decided to take filters with the smallest number of parameters possible and so with very little possibility of detail.

The model was fitted to experimentally determined impulse responses of the transient channel of the visual system. In most cases the model's parameters were found so a reasonable fit was obtained (considering the variance in the measurement data). However, the second negative phase of the impulse response is mostly underestimated by the model. This is a consequence of the rigid choice of the linear filters. Other filters that are slight modifications of the one proposed here may provide a better fit to the impulse response data without losing the concept of the model. Since the concept is considered of more importance than its actual implementation and because probably only slight modifications in the model are sufficient to provide better fits, we continue to examine the behavior of the model.

It was found that under the same conditions (background level and field diameter) the estimated model was essentially the same for different subjects. Comparison of the results from the fits at different background levels showed that from the three parameters in the model only the angular frequency changed by more than the estimated standard deviation. This means that the model also incorporates a very simple description of changes in background level.

Finally, it was shown that the model is in agreement with other psychophysical data. This was shown by predicting the threshold-versus-duration curve and the sensitivity to sinusoidal stimuli. The model's threshold-versus-duration curves agree well with the experimental data. From the prediction of gain characteristic it was found that the low-frequency side is overestimated. This is a direct consequence of the underestimation of the second negative phase of the impulse response rather than a discrepancy. The phase characteristic shows a linear dependence on frequency. This not only agrees with the experimental results, but may also be an attractive system property. In view of the idea of parallel operating channels, linear phase characteristics would provide an easy means to establish timing between transient and sustained channels. Sustained channels (see the impulse response of point sources [7]) also show linear phase behavior over the essential part of their frequency spectrum.
In short, we conclude that a chain of filters incorporating a matched filter is an attractive frame for analyzing and understanding the temporal behavior of the transient channel of the visual system.

\section{APPENDIX}

Consider the output of a linear filter $L$ detected by a deterministic mechanism with threshold $d$. The detection process, however, is stochastic because of the noise source $n(t)$ (see Fig. 10). The noise $n(t)$ is assumed stationary and white. For the mean and the variance $\sigma_{0}^{2}$ of $n(t)$ we take

$$
\begin{gathered}
\varepsilon[n(t)]=0 \\
\varepsilon\left[n(t)^{2}\right]=\sigma_{0}^{2}
\end{gathered}
$$

where $\mathcal{E}[$ ] denotes the expected value. It is assumed that the high threshold assumption is valid, i.e., $\sigma_{0}<<d$. For a pulse to be detected in the deterministic case $\left(\sigma_{0}=0\right)$, a certain amplitude $\epsilon_{1}$ is taken

$$
\epsilon_{1} \Delta t U_{\delta}\left(t_{e x}\right)=d
$$

where $\Delta t$ is the duration of the pulse ( $\Delta t$ much smaller than the time constants of the linear filter), $U_{\delta}(t)$ is the impulse response of the linear filter, and $t_{c x}$ is the time of occurrence of the extremum of the impulse response. In the stochastic case and assuming a high threshold and one clear dominant extremum of $U_{\delta}(t),(18)$ has to be corrected for the noise contribution at $t_{e x}$

$$
\epsilon_{1} \Delta t U_{\delta}\left(t_{e x}\right)=d-n\left(t_{e x}\right) .
$$

For the detection of an impulse response perturbed by an amplitude normalized function $f(t)$ under the same assumptions as earlier, an equation is found similar to (19)

$$
\epsilon_{c}\left\{\Delta t U_{\delta}\left(t_{c x}\right)+q f\left(t_{c x}-\tau\right)\right\}=d-n\left(t_{e x}\right)
$$

where $q$ is a constant, so

$$
q f\left(t_{e x}-\tau\right) \ll U_{\delta}\left(t_{c x}\right), \quad \text { for all } \tau .
$$

$\epsilon_{c}$ will have a stochastic nature too. From (19) and (20) it is found that a constant ratio exists between the mean $\epsilon_{01}$ and $\epsilon_{0 c}$ and the standard deviation $\left(\sigma_{1}\right.$ and $\left.\sigma_{c}\right)$ of $\epsilon_{1}$ and $\epsilon_{\mathrm{c}}$

$$
\frac{\sigma_{1}}{\epsilon_{01}}=\frac{\sigma_{c}}{\epsilon_{0 c}}=\frac{\sigma_{0}}{d}
$$

This ratio is called the Crozier coefficient. This ratio is constant over a large number of stimulus conditions [30]. The normalized disturbance is calculated according to [7]

$$
g\left(t_{e x}-\tau\right)=\frac{f\left(t_{c . x}-\tau\right)}{\Delta t U_{\delta}\left(t_{c . x}\right)}=q^{-1}\left\{\frac{\epsilon_{1}}{\epsilon_{c}(\tau)}-1\right\} .
$$

(In the case that the disturbing signal is an impulse, we get $f\left(t_{e x}-\tau\right)=\Delta t U_{\delta}\left(t_{e x}-\tau\right)$ and $g\left(t_{e x}-\tau\right)=U_{\delta}\left(t_{e x}\right.$ $-\tau) / U_{\delta}\left(t_{e x}\right)=U_{\delta}^{*}\left(t_{e x}-\tau\right)$, i.e., the amplitude normalized impulse response.)

The function $g$ will be stochastic with mean $g_{0}(\tau)$ and variance $\sigma_{g(\tau)}^{2}$. Using a Taylor series expansion around $\epsilon_{01}$ 


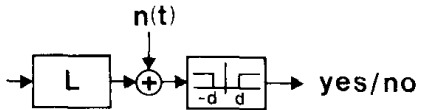

Fig. 10. The linear model $L$ [impulse response $\left.U_{b}(t)\right]$, the additive noise $n(t)$, and the detection mechanism (with threshold $d$ ).

and $\epsilon_{0 c}$, we find that

$$
\begin{aligned}
& g_{0}(\tau) \simeq q^{-1}\left\{\frac{\epsilon_{1}}{\epsilon_{c}}-1\right\}_{\epsilon 01, \epsilon)_{k}}=q^{-1}\left\{\frac{\epsilon_{01}}{\epsilon_{0 c}}-1\right\} \\
& \sigma_{g(\tau)}^{2} \simeq \sigma_{1}^{2}\left(\frac{\partial g}{\partial \epsilon_{1}}\right)_{\epsilon(01, \epsilon \mid)_{c}}^{2}+\sigma_{c}^{2}\left(\frac{\partial g}{\partial \epsilon_{\epsilon}}\right)_{\epsilon \mid 1) . \epsilon|c| c}^{2} \\
& =2\left(\frac{\sigma_{0}}{q d}\right)^{2}\left[1-q g_{0}(\tau)\right]^{2} \\
& \simeq 2\left(\frac{\sigma_{0}}{q d}\right)^{2} \text {. }
\end{aligned}
$$

The last approximation stems from the perturbation requirement (21). From (25) it is seen that at each sampling moment the noise is approximately the same. The measured noise at different moments $\tau$ can therefore be compared directly to each other, and the introduction of a mean measured noise $\sigma_{m}^{2}(13)$ is justified.

\section{REFERENCES}

11] W Reichardt "Autocorrelation A principle for the evaluation of sensory information by the central nervous system," in Sensory Communication, W. A. Rosenblith. Ed. New York: Wiley. 1971.

[2] C. Rashbass, "The visibility of transient changes of luminance," $J$. Physiol., vol. 210, pp. 165-186, 1970.

[3] J. J. Kulikowski and D. J. Tolhurst. "Psychophysical evidence for sustained and transient detectors in human vision," $J$. Physiol., vol. 232, pp. 149-162, 1973

14] J. A. J. Roufs, "Dynamic properties of vision-IV. Thresholds of decremental flashes, incremental flashes and doublets in relation to flicker fushion," Vision Res., vol. 14, pp. 831-851, 1974.

[5] B. G. Breitmeyer and L. Ganz, "Implications of sustained and transient channels for theories of visual pattern masking, saccadic suppression, and information processing." Psychol. Rev., vol. 83 , pp. $1-36,1976$

[6] M. Green, "Masking by light and the sustained-transient dichotomy," Perception and Psychophys., vol. 35. no. 6. pp. 519-539. 1984.

[7] J. A. J. Roufs and F. J. J. Blommaert, "Temporal impulse and step responses of the human eye obtained psychophysically by means of a drift-correcting perturbation technique," Vision Res.. vol. 21, pp. $1203-1221,1981$.

[8] F. J. J. Blommaert and J. A. J. Roufs. "Prediction of thresholds and latency on the basis of experimentally determined impulse responses," Biol. Cybern., vol. 56, pp. 329-344, 1987.

[9] Numerical Algorithms Group Ltd.. Fortran Minimanual, Mark 9 NAG Central Office, Oxford, UK, 1981

[10] A. van den Bos and P. Eykoff. "Model building and parameter estimation as means for intelligent measurements, "presented at the 5th Int. IMEKO Symp. Intell. Measure., Jena. DDR, June 1986.

[11] P. E. Gill, W. Murray, and M. H. Wright. Practical Optimization. London: Academic. 1981

[12] D. H. Kelly and R. E. Savoie, "Theory of flicker and transient re sponses. III. An essential nonlinearity," J. Opt. Soc. Amer., vol. 68. pp. 1481-1490, 1978

113] C. Rashbass, "Unification of two contrasting models of the visua incremental threshold "Vision Res, vol 16,pp 1281-1283,1976.

[14] A. Papoulis, Signal Analysis. New York: McGraw-Hill, 1977.

[15] G. E. Legge, "Sustained and transient mechanisms in human vision: Temporal and spatial properties," Vision Res., vol. 18. pp. 69-81, 1978.

[16] C. A. Burbeck and D. H. Kelly, "Contrast gain measurements and the transient/sustained dichotomy,"J. Opt. Soc. Amer., vol. 71, pp. 1335-1342, 1981 .

[17] J. A. J. Rouls, H. A. L. Piceni, and J. A. Pellegrino van Stuyvenberg, "Phase and gain of the visual transient system," IPO Annu. Prog. Rep., vol. 19, pp. 49-56, 1984.

[18] J. A. J. Roufs. J. A. Pellegrino van Stuyvenberg, and H. A. L. Piceni, "Phase and gain analysis of subthreshold-flicker and flash responses." Perception, vol. 13, p. A15, 1984

$119]$ A. C. den Brinker. "Modelling the transient visual system," Ph.D. dissertation. Eindhoven Univ. Technol., Eindhoven, The Netherlands, 1989.

120] G. L. Turin, "An introduction to matched filters." IRE Trans. Inform. Theor, vol. IT-6, pp. 311-329, 1960.

(21] F. E. W. Vervuurt, "Pseudo-matched filter models for the transient visual system" (in Dutch), Eindhoven Univ. Technol., Eindhoven, Int. Rep. ET-12-88. 1988.

122] D. H. Kelly, "Visual responses to time-dependent stimuli. I. Amplitude sensitivity measurements." J. Opt. Soc, Amer., vol, 51, pp. 422429. 1961

[23] J. A. J. Roufs, "Dynamic properties of vision-I. Experimental relationships between flicker and flash thresholds." Vision Res., vol. 12 , pp. $261-278,1972$.

[24] M. G. F. Fuortes and A. L. Hodgkin. "Changes in time scale and sensitivity in the ommatidia of Limulus," J. Physiol., vol. 172, pp. 239-263. 1964

[25] K.-I. Naka. "The cells horizontal cells talk to," Vision Res., vol. 22 . pp. $653-660,1982$.

[26] H. de Lange, "Relationship between critical flicker frequency and a set of low-frequency characteristics of the eye," J. Opt. Soc. Amer.. vol. 44, pp. $380-389,1954$.

[27] J. Krauskopf. "Discrimination and detection of changes in luminance," Vision Res., vol. 20, pp. 671-677. 1980.

128] Y. Bard, Nonlinear Parameter Estimation. New York: Academic. 1974.

[29] J. R. Wolberg. Prediction Analysis. New York: D. Van Nostrand, 1967.

1301 J A J Roufs. "Dynamic properties of vision-VI. Stochastic threshold fluctuations and their effect on flash-to-flicker sensitivity ratio," Vision Res., vol. 14, pp. 871-888, 1974.

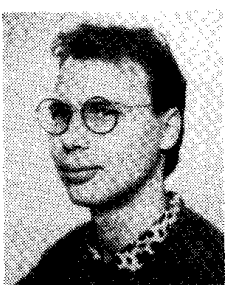

Albertus C. den Brinker received the M.Sc. degree in electrical engineering in 1983 and the $\mathrm{Ph}$.D. degree in 1989 for his work on temporal models of the human visual system, both from the Eindhoven University of Technology. Eindhoven. the Netherlands.

Since that time he has been working in the Interdepartmenta! Group "Retina Models." which is a cooperation between the Institute for Perception Research (IPO). Eindhoven and the Department of Electrical Engineering at the Eindhoven University of Technology. His current research interests are vision and optical computing.

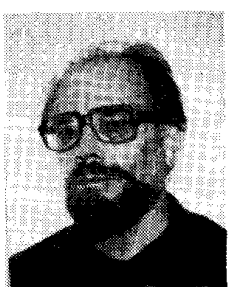

Hans A. L. Piceni was born in Amsterdam, Hol land, on November 20, 1926. He received the $M . S c$. degree in electrical engineering from the Delft University of Technology, Delft. The Netherlands, in 1960.

From 1960 to 1966 he was Head of the Electronical Department in a nuclear physics research institute (IKO/FOM). Thereafter, he was working in the Department of Electrical Engineering, Eindhoven University of Technology with the group of Measurement and Control. His succes sive interests were hybrid computer realization and applications of it, and the model building for the human visual system.

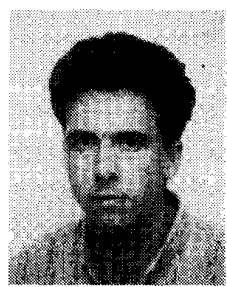

Frans E. W. Vervuurt received the M.Sc de gree in electrical engineering from the Eindhoven University of Technology. Eindhoven, The Neth erlands, in 1988.

He is currently with ATP Netherlands BV where he is working on a research project on tele communications systems. 